\title{
Cyclooxygenase-2 and $\beta$-Catenin as Potential Diagnostic and Prognostic Markers in Endometrial Cancer
}

\author{
Lin Deng ${ }^{1,2 \star}$, Haiyan Liang ${ }^{1 *}$ and Yi Han ${ }^{3}$ \\ ${ }^{1}$ China-Japan Friendship Hospital, Beijing, China, ${ }^{2}$ Graduate School of Peking Union Medical College, Chinese Academy of \\ Medical Sciences, Beijing, China, ${ }^{3}$ Beijing Haidian Center for Disease Control and Prevention, Beijing, China
}

\section{OPEN ACCESS}

Edited by:

Giuseppe Vizzielli,

Agostino Gemelli University

Polyclinic, Italy

Reviewed by:

Martina Borghese,

Hospital Mauritian Turin, Italy

Thangesweran Ayakannu,

University of Liverpool,

United Kingdom

Giorgia Dinoi,

A. Gemelli University Hospital

Foundation, Italy

Serena Cappuccio

Agostino Gemelli University

Polyclinic, Italy

*Correspondence:

Lin Deng

flora1085@163.com

Haiyan Liang

13611311360@139.com

Specialty section:

This article was submitted to

Women's Cancer,

a section of the journal

Frontiers in Oncology

Received: 19 October 2019

Accepted: 13 January 2020

Published: 21 February 2020

Citation:

Deng L, Liang H and Han Y (2020) Cyclooxygenase-2 and $\beta$-Catenin as Potential Diagnostic and Prognostic

Markers in Endometrial Cancer.

Front. Oncol. 10:56

doi: 10.3389/fonc.2020.00056
Objectives: Explore the diagnostic and prognostic value of cyclooxygenase- 2 and wnt3a/ $\beta$-catenin pathway in endometrial cancer.

Methods: A prospective cohort study of 93 women underwent hysterectomy at the China-Japan Friendship Hospital (61 patients with primary endometrial carcinoma, and 32 control patients with uterine prolapse or leiomyoma of uterus). Cox2 and $\beta$-catenin expression were determined by immunohistochemistry. The serum levels of cox2 and wnt3a were detected via ELISA assays.

Results: Patients with endometrial cancer showed overexpression of cox2 and $\beta$-catenin, as well as significantly higher serum levels of cox2 and wnt3a. The serum cox2 level, which is highly significant in predicting the risk of disease progression (RR, 9.617, 95\% confidence interval, 1.162-79.622, $P=0.036$ ), showed good diagnostic and prognostic potential, with cut-off of $55 \mathrm{U} / \mathrm{L}$, but alongside $\beta$-catenin expression in tissues, were related to poor prognosis (RR, 12.426; 95\% confidence interval, 1.618-95.450; $P=0.015)$.

Conclusion: Serum levels of cox2 and wnt3a exhibited diagnostic value for endometrial cancer. Cox2 serum levels and $\beta$-catenin expression also showed potential value of prognostic prediction. Cox2 serum levels might be a potential marker for early diagnosis and prognosis prediction in endometrial cancer.

Keywords: cyclooxygenase-2, $\beta$-catenin, wnt3a, endometrial cancer, prognostic marker, diagnostic marker

\section{INTRODUCTION}

Endometrial cancer (EC), accounting for $20-30 \%$, is one of the three major female genital tract malignancies $(1,2)$. Most of them occurs in women aged 55-65 years, but was recently diagnosed in younger women (2). EC is divided into two types based on its dependence on estrogen, type I-estrogen-dependent cancer and type II-estrogen independent cancer $(2,3)$. Despite extensive research, the overall survival rate for EC patients is yet to improve, with 288,000 new cases diagnosed and 74,000 deaths registered each year worldwide (4). Therefore, identification of clinically prognostic risk factors is crucial to enhance the survival rate of EC patients. 
Recently, some risk factors for EC such as age and genetics, were identified, but the reasons for the occurrence and progression of EC remain elusive (2). Recurrence and metastasis are important steps in the process of cancer progression (5). Several factors, including cyclooxygenase- $2(\operatorname{cox} 2)$ and $\beta$-catenin, are known to be abnormally regulated in $\mathrm{EC}(6,7)$, suggesting their potential relevance to EC prognosis.

Cox2, which is involved in inflammatory processes, has been found to play a key role in prostaglandin biosynthesis $(8,9)$. Generally, the cox 2 protein is only expressed in a limited number of cell types, whereas overexpressed in a variety of malignant tumors, suggesting its possible role in carcinogenesis (6, 10-15). Moreover, several mediatory factors, such as tumor promoters, proinflammatory cytokines and growth factors, have been reported to induce the upregulation of cox2 expression (16). Emerging data have demonstrated that cox 2 is involved in many signaling pathways associated with cell proliferation, metastasis, and drug resistance $(12,13)$. Therefore, cox 2 might be a potential factor affecting EC progression.

The $\beta$-catenin protein, an important intracellular transducer in wnt pathway, plays key roles in the maintenance of tissue homeostasis during adulthood $(4,17)$. Importantly, genetic aberrations have been identified in $\beta$-catenin expressed in EC tissue, suggesting that $\beta$-catenin might be associated with EC progression as well $(7,18,19)$. The wnt/ $\beta$-catenin pathway activates the expression of several important proteins responsible for the cell cycle, proliferation, and survival (20,21). Moreover, $\beta$-catenin, together with E-cadherin, forms adherent junctions that mediate cell adhesion, which is a major factor affecting cell migration and invasion (22-24). Therefore, $\beta$-catenin might constitute a potential factor inducing EC growth and metastasis.

Despite these promising indications, the prognostic role and mechanisms of action of $\operatorname{cox} 2$ and $\beta$-catenin in EC have been widely disputed. Yet, to our knowledge, their impact on EC responses to chemotherapy and EC recurrence rates have never been evaluated. In this study, we applied immunohistochemical analysis and ELISA to compare the expression of cox 2 and wnt pathway-related proteins in EC and normal endometrial tissues. We aimed to investigate the relationships of $\operatorname{cox} 2$ and $\beta$ catenin expression with various clinicopathological factors and then identify and delineate the potential mechanisms involved.

\section{MATERIALS AND METHODS}

\section{Patients}

Patients were enrolled from December 2016 to December 2018 at the Department of Obstetrics and Gynecology, China-Japan Hospital in Beijing, China. In this prospective cohort study, we included 93 patients who underwent hysterectomy. Among these patients, 61 patients (age ranged from 41 to 78 years old) had primary EC and 32 patients (age ranged from 43 to 74 years

Abbreviations: cox2, cyclooxygenase 2; wnt3a, Wnt family member 3A; EC, endometrial cancer; ROC, receiver operating characteristic; PFS, progression free survival; RR, Relative Risk; OR, Odds ratio; AUC, area under the curve; CA125, cancer antigen 125; HE4, human epididymis protein; IHC, immunohistochemistry; NSAIDs, non-steroidal anti-inflammatory drugs; HRP, horseradish peroxidase; $\mathrm{H} \& \mathrm{E}$, hematoxylin-eosin staining. old) had normal endometrium but underwent hysterectomy due to uterine prolapse or leiomyoma of the uterus. The study was approved by the Medical Ethics Committee of the China Japan Friendship Hospital. All participants gave their informed consent before being involved in the study. Histopathological results were evaluated by two independent gynecological pathologists (25). In each case, clinical information, such as age, presence of hypertension, BMI, and type 2 diabetes were collected, and image characteristics and pathological information were also obtained.

Inclusion criteria: complete clinical data; aged 40-80 years; histopathological diagnosis of endometrioid EC; without having received surgery, radiotherapy, chemotherapy or hormone therapy before enrollment; without carcinoma in other organs; without coagulation disorders; without endometriosis; without acute or chronic inflammation; without a history of taking non-steroidal anti-inflammatory drugs (NSAIDs); provision of a written consent form. Additionally, individuals with a history of endometrial polyp or endometrial atypical hyperplasia were excluded from the control group.

Serum samples obtained before surgery were stored at $-80^{\circ} \mathrm{C}$ alongside collected tissue specimens and kept until molecular studies were performed. Tissue samples were obtained after the surgery and divided into pieces. Some were frozen at $-80^{\circ} \mathrm{C}$, and others were prepared for immunohistochemical (IHC) studies by fixation in $10 \%$ buffered formaldehyde solution for $48 \mathrm{~h}$, routine dehydration, immersion in paraffin for embedding, and continuous slicing into $4-\mu \mathrm{m}$-thick sections according to standard procedures.

\section{Immunohistochemistry}

Two biological markers ( $\operatorname{cox} 2$ and $\beta$-catenin) were investigated via IHC study: cox2, $\beta$-catenin. Cox2 was assessed using monoclonal Ab (Rabbit Anti-cox2, ab15191, abcam, 1:200) and $\beta$-catenin was detected with a monoclonal Ab (Rabbit Anti- $\beta$ catenin, 8480, cst, 1:200). All the secondary antibodies and DAB stain were purchased from Beijing Zhongshan Golden Bridge Biotechnology Co., Ltd.

Deparaffinized sections by xylene and subsequently sequential immersion hydrated them by graded alcohol solutions (5). Antigen unmasking was performed in a container with TrisEDTA buffer at $\mathrm{PH}$ 9.0, 0.5\% Tween 20 using heat treatment in a microwave oven $(800 \mathrm{~W}$ for $2 \mathrm{~min}$ without the sections and then turn to $150 \mathrm{~W}$ for $10 \mathrm{~min}$ with the sections). Sections were allowed to cool in the buffer for $30 \mathrm{~min}$ at $25^{\circ} \mathrm{C}$. And then, rinsed them in phosphate buffer saline (PBS), 5 min each for three times. Next, incubated sections were treated with $3 \%$ hydrogen peroxide for $15 \mathrm{~min}$ at room temperature $\left(25^{\circ} \mathrm{C}\right)$ to block the activity of endogenous peroxidase; this was followed by three washes with PBS and incubation with goat serum at $25^{\circ} \mathrm{C}$ for $40 \mathrm{~min}$ (5). Blocking reagent was removed, and sections were, then, probed with different monoclonal antibodies or with PBS (negative control) for $2 \mathrm{~h}$ at $25^{\circ} \mathrm{C}$ or overnight at $4^{\circ} \mathrm{C}$. Sections were incubated with primary antibodies diluted with $\mathrm{PBS}$ and $1.5 \%$ bovine serum albumin (BSA). After primary antibody incubation, the sections were washed 3 times with PBS. Then, sections were incubated for $15 \mathrm{~min}$ with biotin-labeled secondary antibody. Washed with PBS for three 
times. Incubated sections with horseradish peroxidase (HRP)labeled streptomycin working stain for $15 \mathrm{~min}$, and washed 3 times with PBS (5). Finally, the sections were stained using diaminobenzidine as a chromogen and Meyers hematoxylin for counterstaining, then, mounted.

\section{Evaluation of Immunostaining}

Immunostaining results were evaluated by two blinded pathologists using a light microscope, and a combined score based on the percentage of cells stained and staining intensity was used for assessment. Immunohistochemical cox 2 and $\beta$-catenin staining were scored according to the criteria indicated by Krajewska et al. (26) and Kahane et al. (27). Immunohistochemically positive cells were considered as cells with membranes or cytoplasm containing yellow or brown granules. According to the percentage of immunohistochemically positive cells, the sections were graded as follows: 0 , negative; $1,1-25 \%$ positive cells; $2,26-50 \%$ positive cells; $3,51-75 \%$ positive cells; and 4, 76-100\% positive cells (5). Immunostaining intensity was evaluated by the shade of brown granules and rated as follows: 0 , negative; 1 , weakly positive; 2 , moderately positive; and 3 , intensely positive (5). Both score sets were multiplied to derive composite points as follows: 0 point $(-) ; 1-4$ points $(+)$; $5-8$ points $(++)$; and $9-12$ points $(+++)$. A combined staining score larger than or equal to 5 was considered as indicative of positive staining. The final score for each specimen was obtained by averaging the rating scores given by the two pathologists. If the scores given by both experts differed by more than 3 points, the evaluation would be repeated.

\section{ELISA Assay}

ELISA was performed using a kit, according to the manufacturer's instructions. Firstly, set standard and experimental sample wells. Secondly, added $50 \mu \mathrm{l}$ of standard solution to each standard wells and $50 \mu \mathrm{l}$ combination $(10 \mu \mathrm{l}$ testing sample and $40 \mu \mathrm{l}$ sample diluent) to the testing sample wells. Blank wells were left empty. Thirdly, added HRP-conjugated reagent $100 \mu l$ to each well and mixed. Next, covered the plate with an adhesive strip, and then, incubated at $37^{\circ} \mathrm{C}$ for $60 \mathrm{~min}$. Washed the plate with washing solution for four times and then, inverted and blotted it on a clean paper (28). Subsequently, added chromogen solution A and B 50 $\mu l$ in each well respectively, mixed, and incubated at $37^{\circ} \mathrm{C}$ in dark place for $15 \mathrm{~min}$ after mixing. Finally, added $50 \mu \mathrm{l}$ stop solution to each well and measured optical density with microtiter plate reader at $450 \mathrm{~nm}$ within $15 \mathrm{~min}(29,30)$.

\section{Statistical Analysis}

Data were analyzed utilizing the SPSS 25.0 software and values were presented as average value \pm standard deviation. Two groups' comparison: independent sample $t$-test or MannWhitney test. Comparisons of more than two groups: ANOVA analysis or non-parametric Kruskal-Wallis H tests. Chi-square test was used to compare rates (25). Receiver operating characteristic (ROC) curves using to evaluate the sensitivity and specificity of biomarkers in diagnosis. Spearman's and Pearson's correlation coefficients and logistic regression model were estimated to determine correlation (25). Kaplan-Meier and
Cox regression methods were used to illustrate Progression Free Survival (PFS) and the relative risk (RR) for patients with normal biomarkers levels vs. elevated biomarkers levels. $P$-value $<0.05$ was considered to have a statistically significance.

\section{RESULTS}

\section{Clinical Characteristics}

The case group comprised 61 patients with EC, with a mean age of 58 years old (range from 41 to 78 years old), and a mean BMI of $24.28 \mathrm{~kg} / \mathrm{m}^{2}$ (range from 18.61 to $30.12 \mathrm{~kg} / \mathrm{m}^{2}$ ). The control group comprised 32 patients with leiomyoma of the uterus or uterine prolapse, with a mean age of 56 years (range from 43 to 74 years) and mean BMI of $24.62 \mathrm{~kg} / \mathrm{m}^{2}$ (range from 18.22 to $30.12 \mathrm{~kg} / \mathrm{m}^{2}$ ). There were no statistically significant differences in age distribution and BMI between the two groups $(P>0.05)$. No significant differences were observed in menopausal status, cesarean history, dysmenorrhea history, diabetes, and arterial hypertension as well $(P>0.05)$ (Table 1 ).

Based on the classification by the Federation International of Gynecology and Obstetrics (FIGO), 26 (42.62\%) patients had stage I, 19 (31.15\%) had stage II, 14 (22.95\%) had stage III, and $2(3.28 \%)$ had stage IV EC. Among all patients, 38 $(62.3 \%)$ had $<1 / 2$ myometrial invasion, and $23(37.7 \%)$ had $>1 / 2$ myometrial invasion. Tumors were highly differentiated in $29(47.54 \%)$ patients, moderately differentiated in $19(31.15 \%)$ patients, and poorly differentiated in $13(21.31 \%)$ patients. Ten (16.39\%) patients had tumor-positive lymph nodes, whereas 51 (83.61\%) patients had tumor-negative lymph nodes. Thirty-eight (62.3\%) patients did not have vessel invasion, whereas $23(37.7 \%)$ had (Table 2). The mean follow-up time was $24 \pm 4.2$ months (range from 13 to 33 months), and no patients died because of EC till now. However, 20 (32.8\%) patients showed disease progression, such as recurrence, metastasis, or drug resistance (Table 2).

\section{Expression of Cox 2 and $\beta$-Catenin in EC Tissues}

In EC epithelial cells cytoplasm, intensely positive cox2 expression was observed: brown granules were diffused in the cytoplasm or linearly along the nucleus. Similarly, $\beta$-catenin was expressed in the cytoplasm or even, in the nucleus. Llittle or no immunoreactivity for $\operatorname{cox} 2$ and $\beta$-catenin were observed in normal endometrium. Cox 2 and $\beta$-catenin positively expression were defined as tumor with a score of at least 5. Figure 1 shows a representative image of EC tissue with intensely immunoreactivity for both cox 2 and $\beta$-catenin.

\section{Relationship Between Cox2 or $\beta$-Catenin Expression and Clinicopathological Parameters}

Among 61 patients with EC, 55 (90.2\%) exhibited a positive expression for cox 2, whereas 47 (77\%) patients showed positivity for expression for $\beta$-catenin. Protein expression of cox 2 and $\beta$-catenin was associated with the FIGO stages, differentiation $(P<0.05)$ but not related to lymph node metastasis and 
TABLE 1 | Detailed clinical characteristics of the study participants.

\begin{tabular}{|c|c|c|c|}
\hline & $\begin{array}{l}\text { Control, } \\
n=32(100 \%)\end{array}$ & $\begin{array}{l}\text { Case, } \\
n=61(100 \%)\end{array}$ & $P$-values \\
\hline \multicolumn{4}{|l|}{ Age } \\
\hline Pre-menopausal & $14(43.75 \%)$ & $23(37.70 \%)$ & NS \\
\hline Post-menopausal & 18 (56.25\%) & $38(62.30 \%)$ & \\
\hline NA & $0(0 \%)$ & $0(0 \%)$ & \\
\hline \multicolumn{4}{|l|}{ BMI } \\
\hline$\leq 24.9$ & $17(53.13 \%)$ & $36(59.02 \%)$ & NS \\
\hline$>24.9$ & $15(46.87 \%)$ & 25 (40.98\%) & \\
\hline NA & $0(0 \%)$ & $0(0 \%)$ & \\
\hline \multicolumn{4}{|l|}{ Diabetes } \\
\hline Yes & $0(0 \%)$ & $0(0 \%)$ & NS \\
\hline No & $32(100 \%)$ & $61(100 \%)$ & \\
\hline NA & $0(0 \%)$ & $0(0 \%)$ & \\
\hline \multicolumn{4}{|l|}{ Arterial hypertension } \\
\hline Yes & $4(12.50 \%)$ & 10 (16.39\%) & NS \\
\hline No & $28(87.50 \%)$ & $51(83.61 \%)$ & \\
\hline NA & $0(0 \%)$ & $0(0 \%)$ & \\
\hline \multicolumn{4}{|l|}{ Cesarean history } \\
\hline Yes & $6(18.75 \%)$ & $8(13.11 \%)$ & NS \\
\hline No & $26(81.25 \%)$ & $53(86.89 \%)$ & \\
\hline NA & $0(0 \%)$ & $0(0 \%)$ & \\
\hline \multicolumn{4}{|l|}{ Dysmenorrhea history } \\
\hline Yes & $3(9.38 \%)$ & $6(9.84 \%)$ & NS \\
\hline No & 29 (90.62\%) & $55(90.16 \%)$ & \\
\hline NA & $0(0 \%)$ & $0(0 \%)$ & \\
\hline \multicolumn{4}{|l|}{ Pregnancy history } \\
\hline Yes & 31 (96.87\%) & 49 (80.33\%) & 0.03 \\
\hline No & $1(3.13 \%)$ & $12(19.67 \%)$ & \\
\hline NA & 0 (0\%) & $0(0 \%)$ & \\
\hline
\end{tabular}

NA, not available; NS, not significant.

vessel invasion $(P>0.05)$. $\beta$-catenin expression was also associated with myometrial invasion depth of tumors $(P<$ $0.05)$. Differences in the expression of $\operatorname{cox} 2$ or $\beta$-catenin expression with clinicopathological parameters are shown in Table 2. Table S1 presents the Spearman rank correlation indices indicating a correlation between the expression of cox 2 and $\beta$-catenin $(P<0.05)$.

\section{Relationship Between Cox2 or wnt3a Serum Levels and Clinicopathological Parameters}

Serum levels of $\operatorname{cox} 2$ and wnt3a were significantly different between case and control patients. Serum levels of wnt3a was associated with the myometrial invasion depth of tumors and vessel invasion $(P<0.05)$. Serum level of cox2 was associated with the tumor differentiation $(P<0.05)$ changes in cox2 and wnt3a serum levels with clinicopathological parameters are shown in Table 3. Nonetheless, cox 2 serum levels were positively correlated with wnt3a levels. Spearman rank correlation indices
TABLE 2 | Association of cox2, $\beta$-catenin of the 61 patients with endometrial cancer.

\begin{tabular}{|c|c|c|c|c|c|c|c|}
\hline \multirow[t]{2}{*}{ Item } & \multirow[t]{2}{*}{$N$} & \multicolumn{3}{|c|}{ Cox2 positive } & \multicolumn{3}{|c|}{$\beta$-catenin positive } \\
\hline & & $N$ & $x^{2}$ & $\boldsymbol{P}$ & $N$ & $X^{2}$ & $P$ \\
\hline \multicolumn{2}{|l|}{ FIGO stage } & & 9.136 & 0.028 & & 10.302 & 0.016 \\
\hline I & 26 & 21 & & & 15 & & \\
\hline$\|$ & 19 & 19 & & & 17 & & \\
\hline III & 14 & 14 & & & 13 & & \\
\hline IV & 2 & 2 & & & 2 & & \\
\hline \multicolumn{2}{|c|}{ Degree of differentiation } & & 7.931 & 0.019 & & 10.649 & 0.005 \\
\hline G1 & 29 & 24 & & & 18 & & \\
\hline G2 & 19 & 19 & & & 16 & & \\
\hline G3 & 13 & 13 & & & 13 & & \\
\hline \multicolumn{2}{|c|}{ Myometrial invasion } & & 5.000 & 0.082 & & 8.787 & 0.012 \\
\hline$<1 / 2$ & 38 & 33 & & & 26 & & \\
\hline$\geq 1 / 2$ & 9 & 9 & & & 7 & & \\
\hline Full-thickness & 14 & 14 & & & 14 & & \\
\hline \multicolumn{2}{|c|}{ Lymph node metastasis } & & 1.876 & 0.171 & & 1.316 & 0.251 \\
\hline Negative & 51 & 46 & & & 38 & & \\
\hline Positive & 10 & 10 & & & 9 & & \\
\hline \multicolumn{2}{|l|}{ Vessel invasion } & & 0.792 & 0.398 & & 2.180 & 0.140 \\
\hline Negative & 38 & 34 & & & 27 & & \\
\hline Positive & 23 & 22 & & & 20 & & \\
\hline
\end{tabular}

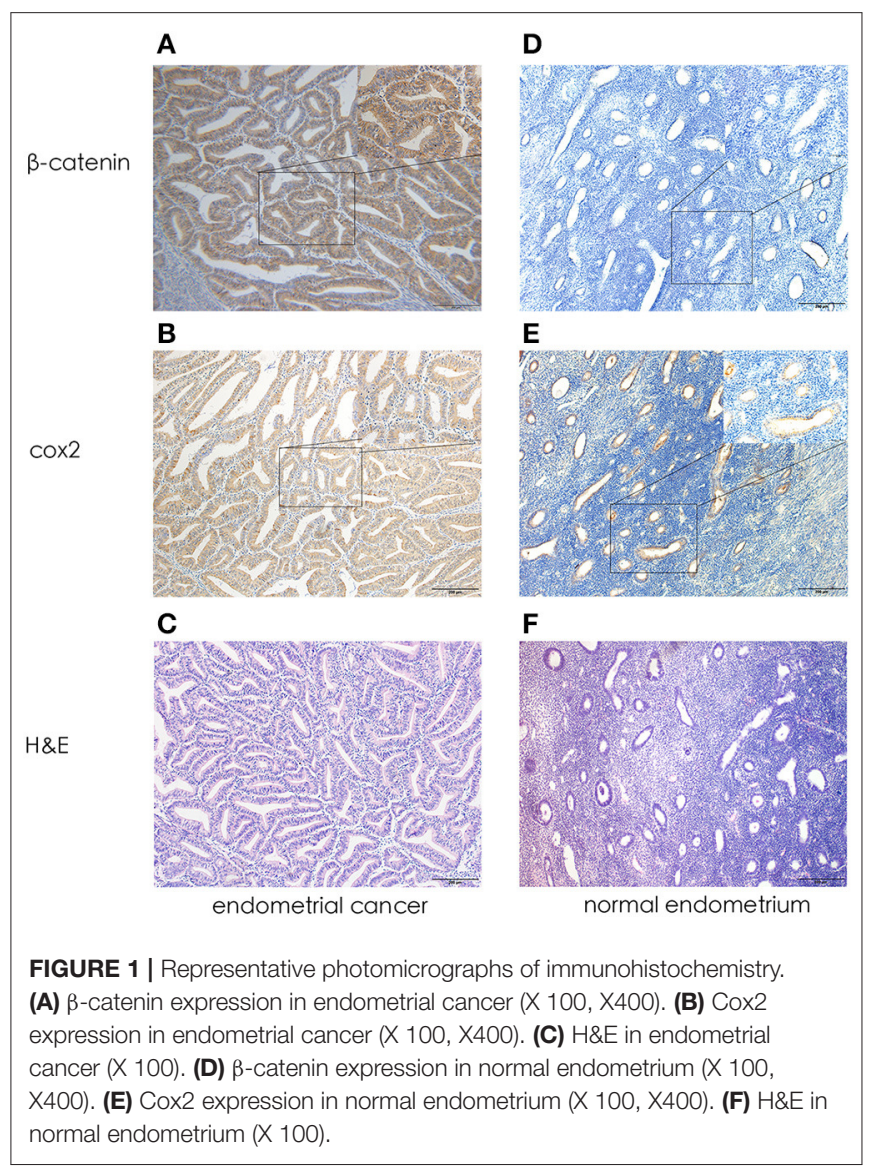


TABLE 3 | Association of cox2, wnt3a serum levels of the 61 patients with endometrial cancer.

\begin{tabular}{|c|c|c|c|c|c|c|c|}
\hline \multirow[t]{2}{*}{ Item } & \multirow[t]{2}{*}{$N$} & \multicolumn{3}{|c|}{ Cox2 > 55 U/L } & \multicolumn{3}{|c|}{ Wnt3a > 25 ng/ml } \\
\hline & & $N$ & $X^{2}$ & $\boldsymbol{P}$ & $N$ & $x^{2}$ & $P$ \\
\hline \multicolumn{2}{|l|}{ FIGO stage } & & 6.034 & 0.110 & & 1.074 & 0.783 \\
\hline I & 26 & 21 & & & 21 & & \\
\hline$\|$ & 19 & 15 & & & 15 & & \\
\hline III & 14 & 14 & & & 12 & & \\
\hline IV & 2 & 2 & & & 2 & & \\
\hline \multicolumn{2}{|c|}{ Degree of differentiation } & & 6.206 & 0.045 & & 5.959 & 0.051 \\
\hline G1 & 29 & 22 & & & 22 & & \\
\hline $\mathrm{G} 2$ & 19 & 17 & & & 15 & & \\
\hline G3 & 13 & 13 & & & 13 & & \\
\hline \multicolumn{2}{|c|}{ Myometrial invasion } & & 1.257 & 0.533 & & 7.490 & 0.024 \\
\hline$<1 / 2$ & 38 & 31 & & & 28 & & \\
\hline$\geq 1 / 2$ & 9 & 8 & & & 8 & & \\
\hline Full-thickness & 14 & 13 & & & 14 & & \\
\hline \multicolumn{2}{|c|}{ Lymph node metastasis } & & 3.515 & 0.061 & & 0.587 & 0.443 \\
\hline Negative & 51 & 42 & & & 41 & & \\
\hline Positive & 10 & 10 & & & 9 & & \\
\hline \multicolumn{2}{|l|}{ Vessel invasion } & & 1.150 & 0.283 & & 5.542 & 0.019 \\
\hline Negative & 38 & 31 & & & 28 & & \\
\hline Positive & 23 & 21 & & & 22 & & \\
\hline
\end{tabular}

between cox2 and wnt3a serum levels were showed in Table S2.

\section{Expression of Cox2 and $\beta$-Catenin in Tissues Is Associated With Patient Diagnosis and Progression-Free Survival (PFS) Time}

Tissue expression of $\operatorname{cox} 2$ and $\beta$-catenin indicated their prognostic potential in terms of vessel invasion, the depth of myometrial invasion and disease progress. Expression of cox 2 differed significantly when patients were stratified according to vessel invasion, myometrial invasion status, and prognosis status, each with an area under the curve (AUC) of 0.702 (sensitivity of $73.9 \%$ and specificity of $63.2 \%$ ), 0.732 (sensitivity of $82.6 \%$ and specificity of $50 \%$ ), 0.789 (sensitivity of $85 \%$ and specificity of $65.9 \%$ ), respectively. For $\beta$-Catenin, the AUC was 0.763 (sensitivity of $78.3 \%$ and specificity of $52.6 \%$ ) for vessel invasion, 0.692 (sensitivity of $78.3 \%$ and specificity of $52.6 \%$ ) for depth of myometrial invasion, and 0.869 (sensitivity of $85 \%$ and specificity of $85.4 \%$ ) for prognosis status. The ROC curves according to lymph node metastasis did not have statistically significance for $\operatorname{cox} 2$ and $\beta$-Catenin expression. Receiver operating characteristics (ROC) curves were shown in Figure 2 and the index of ROC curves are showed in Table S3.

Logistic regression models were constructed to predict the relationship between EC diagnosis and the expression of cox 2 and $\beta$-catenin. Regarding disease diagnosis, our logistic model showed an odds ratios (OR) of 41.840 (95\% confidence interval, 4.750-368.550, $P=0.001)$ for $\beta$-Catenin, and $14.327(95 \%$ confidence interval, 3.419-60.029, $P=0$ ) for cox2. Results from
Kaplan-Meier survival analysis showed that both $\operatorname{cox} 2$ and $\beta$ catenin expression were significantly associated with patients' PFS, which means the survival time without recurrence, death, drug-resistance or metastasis. Take $\beta$-catenin and cox 2 together in the Cox proportional hazard model, the expression of $\beta$ catenin had statistically significance on patients' prognosis $(\beta$ Catenin: RR, 12.426; 95\% confidence interval, 1.618-95.450; $P$ $=0.015)$, suggesting its potential as a single risk factor to EC prognosis (Figure 3).

\section{Serum Level of Cox2 Might Be Good Diagnostic and Prognostic Factor for EC}

Serum levels of cox2 and wnt3a showed the prediction potential in EC diagnosis (cox2: AUC of 0.887 , sensitivity of $95.1 \%$ and specificity of $71.9 \%, P=0$; wnt $3 a$ : AUC of 0.931 , sensitivity of $96.7 \%$ and specificity of $81.2 \%, P=0$ ). Serum levels of cox 2 and wnt3a also showed the prognostic potential in vessel invasion (cox2: AUC of 0.696 , sensitivity of $73.9 \%$ and specificity of $63.2 \%, P=0.011$; wnt3a: AUC of 0.757 , sensitivity of $78.3 \%$ and specificity of $63.2 \%, P=0.001$ ) and lymph node metastasis (cox2: AUC of 0.732 , sensitivity of $80 \%$ and specificity of $62.7 \%, P=$ 0.021 ; wnt3a: AUC of 0.711 , sensitivity of $70 \%$ and specificity of $76.5 \%, P=0.036$ ) and poor prognosis ( $\operatorname{cox} 2$ : AUC of 0.752 , sensitivity of $80 \%$ and specificity of $73.2 \%, P=0.002$; wnt 3 a: AUC of 0.711 , sensitivity of $75 \%$ and specificity of $58.5 \%, P=0.008$ ). ROC curves were shown in Figure 2 and the index of ROC curves are shown in Table S3.

Logistic regression models were built to predict the relationship between serum levels of $\operatorname{cox} 2$ or wnt3a and EC diagnosis. The logistic regression model showed an OR of 10.551 (95\% confidence interval, 2.708-41.111, $P$ $=0.001)$ for wnt3a, and 20.076 (95\% confidence interval, $5.068-79.526, P=0.000)$ for $\operatorname{cox} 2$. The $\operatorname{cox} 2$ and wnt3a serum levels also showed meaningful influence on the PFS of EC patients. Results obtained from Kaplan-Meier survival analysis showed that both cox 2 and wnt3a serum levels were significantly associated with patient survival $(P<0.05)$. Take wnt3a and cox2 levels together in the Cox proportional hazard model with cut-off values of $55 \mathrm{U} / \mathrm{L}$ for cox2 and $25 \mathrm{ng} / \mathrm{ml}$ for wnt3a. Serum levels of cox 2 showed a statistically significant influence on PFS and suggested as a single risk factor (cox2: RR, 9.617, 95\% confidence interval, 1.162-79.622, $P=$ 0.036) (Figure 3).

\section{DISCUSSION}

Curettage and pathohistological examinations are still regarded as the gold standard for EC diagnosis due to the absence of noninvasive biomarkers for predicting the presence and extent of the disease (25). Surgery and chemotherapy or radiotherapy after surgery remain the major treatment options for EC. Recurrence and metastasis are the major causes of EC patient death. Thus, early detection using diagnostic and prognostic biomarkers, thus, has important clinical implications as these could help screen asymptomatic women and distinguish patients with a high risk of disease progression from those with better prognosis. However, 

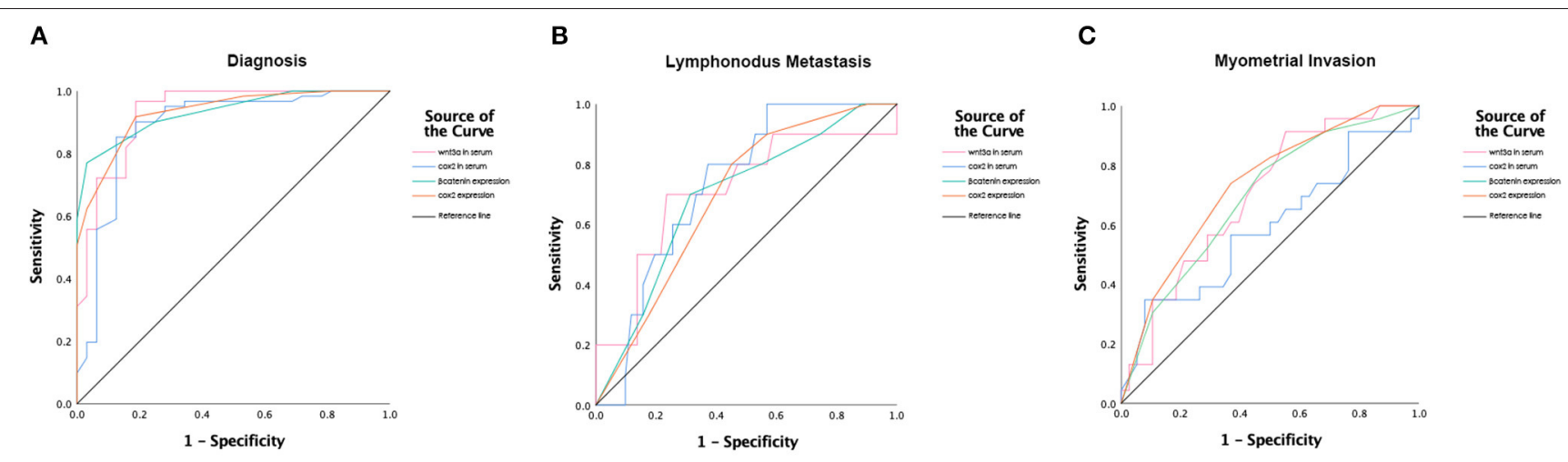

D
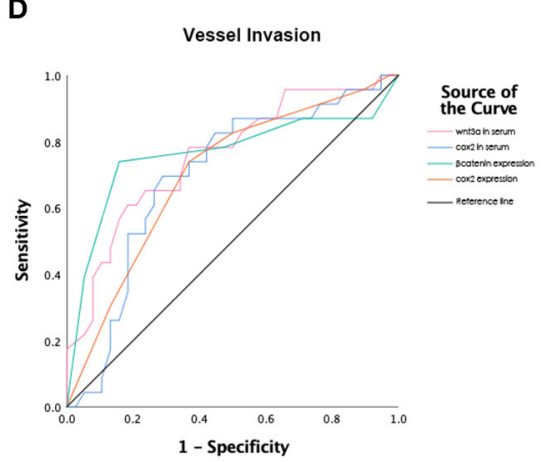

E

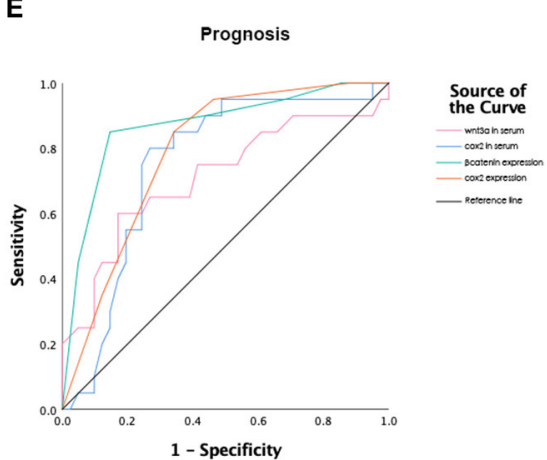

FIGURE 2 | Receiver operating characteristic (ROC) curves for (A) Diagnosis; (B) Prognostic values of lymph node invasion; (C) Prognostic values of myometrial invasion; (D) Prognostic values of vessel invasion; (E) Poor prognostic values.

the number of studies on the exploration of biomarkers that could help early diagnosis and predict the prognosis of patients with EC is still limited. Although there have been several studies in EC patients examining serum indices, such as cancer antigen 125 (CA125), human epididymis protein (HE4), and VEGF (25, 31-33), their reliability and repeatability still lack consensus.

In recent years, several studies have explored the relationship between cox 2 expression and patients' prognosis in EC. However, to our knowledge, none of them have evaluated the serum levels of cox 2 in patients with EC. The current study is the first to detect and measure serum levels of cox 2 and wnt $3 \mathrm{a}$ in EC patients using ELISA, and is the first to show that IHC expression and serum levels of biomarkers correlated with the clinical outcome of EC (34). In this cohort study, we examined cox 2 and wnt3a serum levels using ELISA and tissue expression using IHC. The results showed that $\operatorname{cox} 2$ and wnt $3 a$ serum concentrations correlated with diagnosis, prognosis, and disease extent factors. Similarly, immunochemical analysis revealed that overexpression of cox2 and $\beta$-catenin is associated with EC prognosis. Subsequently, Cox regression models showed that cox 2 serum level and $\beta$-catenin expression were single risk factor to EC prognosis, suggesting that the cox 2 serum level might be used as a potential biomarker for early non-invasive diagnosis and further prognosis prediction of EC. Additionally, $\beta$-catenin expression might be another assistant marker for further prognosis prediction.
Cox2 expression in normal tissues is limited; however, when stimulated by cytokines, growth factors, and oncogenes, its expression is upregulated (16). Notably, cox2 is known to play a key role in the development of various tumor types $(9-12,14)$. Previous studies have indicated that cox 2 could participate in the processes of cell proliferation, migration, and invasion, thus playing a key role in tumor growth and metastasis $(12,14,15)$. However, studies investigating the relationship between cox2 and EC are still limited, and cox 2 serum levels in patients with EC have never been reported. In the present study, we could not detect or only detected very low cox 2 expression in normal endometrial tissues, whereas in EC tissues, $90.2 \%$ positive cox 2 expression was reported. Patients with cox 2 overexpression were more likely to have poor prognosis. Moreover, overexpression of $\operatorname{cox} 2$ also showed relationships with factors related to EC progression, such as FIGO stage, differentiation and myometrial invasion $(P<0.05$ for all), which were similarly to previous studies $(6,10,34)$. Additionally, we evaluated the cox2 serum levels and observed that they were significantly elevated in patients with EC compared to those with normal endometrium. The present results also indicated that cox 2 serum levels can help differentiate among patients with poorly differentiation and prognosis, suggesting its potential prognostic value. Importantly, our study showed that cox2 serum levels with a cut-off value of $55 \mathrm{U} / \mathrm{L}$ were a better predictor of early EC diagnosis, 


\section{A}

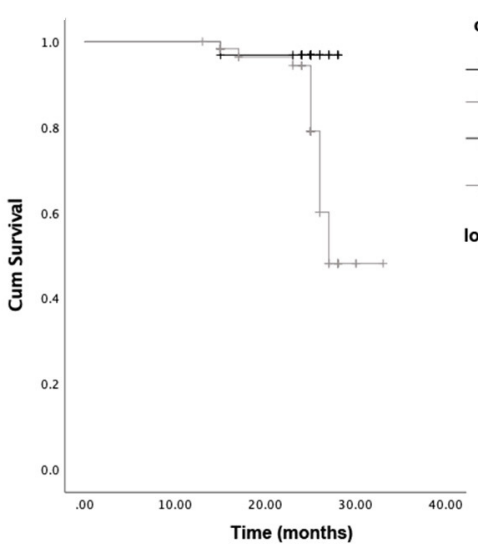

C

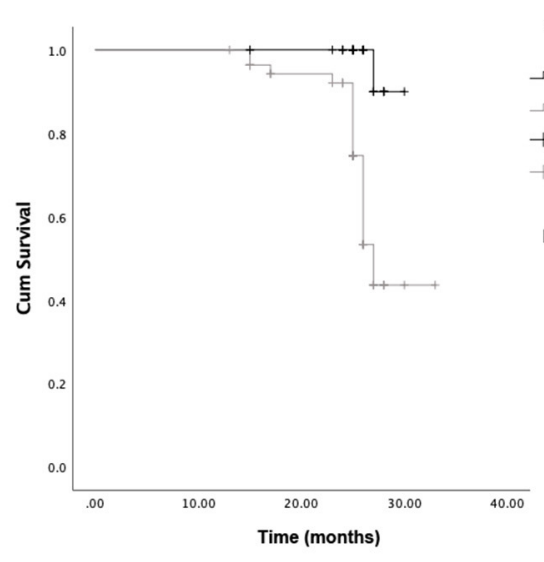

E

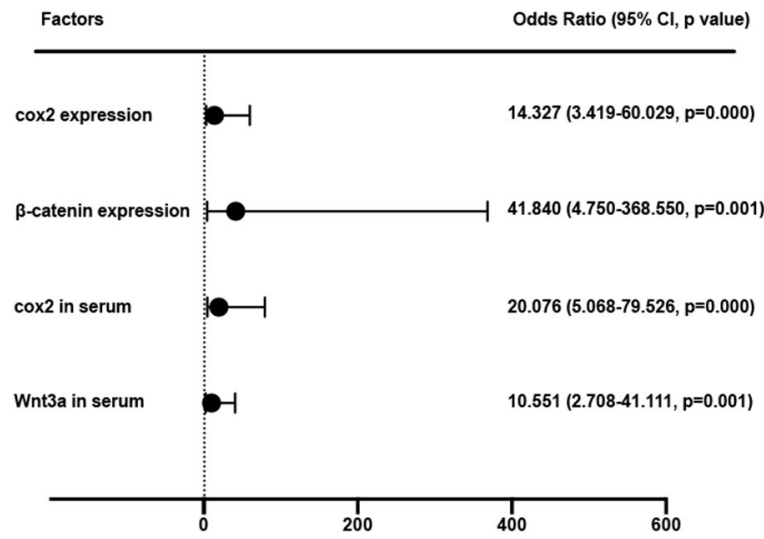

B

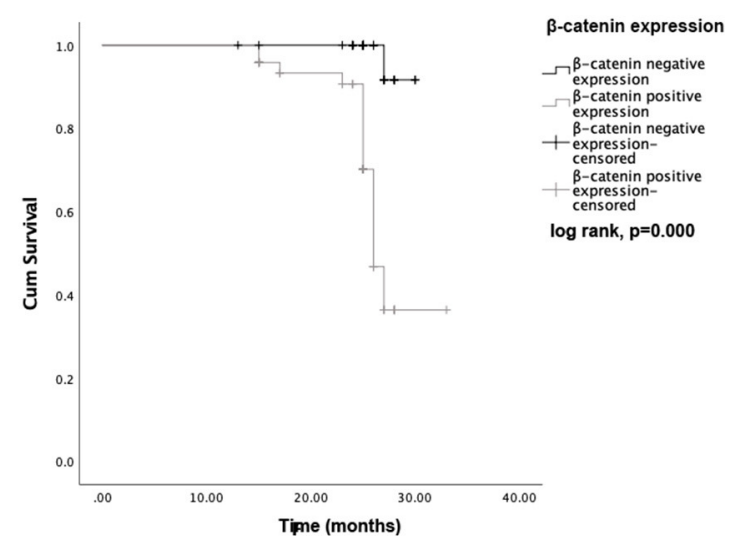

D

Progression Free Survival (PFS)

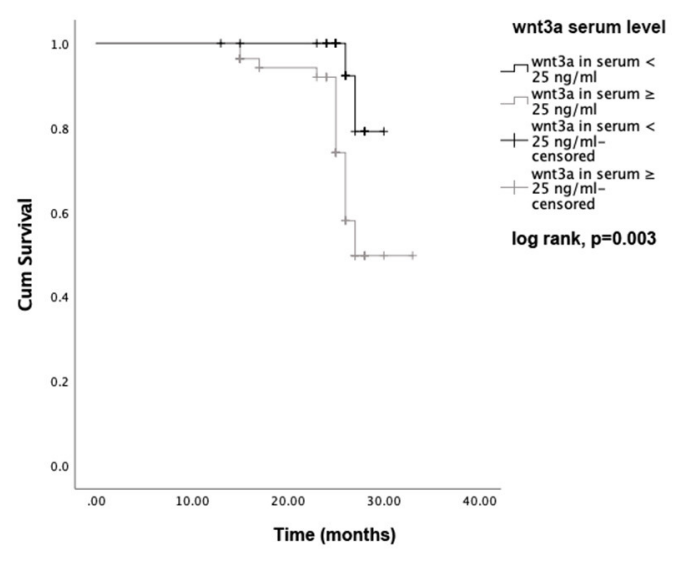

$\mathbf{F}$

Prognosis

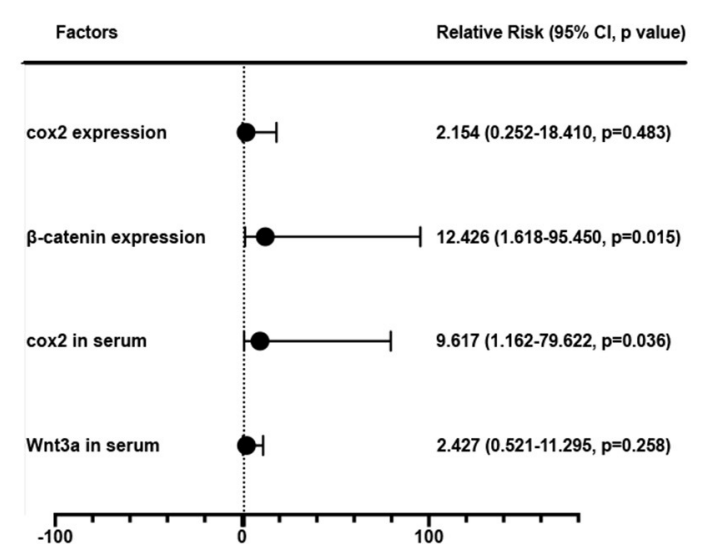

FIGURE 3 | Kaplan-Meier curves for progression-free survival (PFS) and hazard ratios for tumor progression. (A) Comparison of PFS between cox2 positive group and the negative group. (B) Comparison of PFS between $\beta$-catenin positive group and negative group. (C) Comparison of PFS in endometrial cancer patients between those with cox2 serum levels $<55 \mathrm{U} / \mathrm{L}$ and those with cox2 serum levels $\geq 55 \mathrm{U} / \mathrm{L}$. (D) PFS in patients with wnt3a serum levels $<25 \mathrm{ng} / \mathrm{ml}$ vs patients with wnt3a serum levels $\geq 25 \mathrm{ng} / \mathrm{ml}$. (E) Odds ratio (OR) for diagnosis. (F) Relative Risk (RR) for disease progression. For diagnosis and prognosis prediction, cox2 serum level and $\beta$-catenin expression demonstrated a predictive ability.

depth of myometrial invasion, lymph node involvement, vessel invasion, and further prognosis. Kaplan-Meier analysis and Cox regression indicated that patients with higher serum levels of cox2 were more likely to have poor prognosis, such as recurrence, metastasis, and drug-resistance. Cox regression model also indicated cox2 serum level as a single risk factor for EC patients 
(RR, 9.617, 95\% confidence interval, 1.162-79.622, $P=0.036$ ), and was more specific and sensitive compared to the cox 2 tissue expression (RR, 2.154; 95\% confidence interval, 0.252-18.410, $P=0.483$ ), indicating that cox 2 serum levels might potentially be a more efficient marker for early diagnosis and prognosis prediction in high risk EC patients.

Wnt signaling, a cellular pathway pivotal for embryonic development and tissue homeostasis, was found to be effective in primary axis formation, organogenesis, stem cell proliferation, and cell fate decisions as well (35-40). Its aberrant activation has been associated with the majority of human malignancies, including EC $(41,42)$. In particular, $\beta$-catenin is the primary mediator of the oncogenic effect in this signaling pathway $(17,21)$. Almost $30-85 \%$ of type I EC cases show nuclear accumulation of $\beta$-catenin in the epithelium (43-45). Thus, it is important to understand the role of $\mathrm{wnt} / \beta$-catenin signaling in EC. A recent study reported that wnt $/ \beta$-catenin pathway dysregulation to be an important factor in young EC patients with poor prognosis (45-47). Genetic mutations of the wnt $/ \beta$ catenin signaling pathway members are mostly detected in patients with more aggressive histologic type of EC and are related to worse prognosis and poor overall survival rate $(4,10$, $45)$. These findings are similar to the results of our study. In this study, we detected the wnt 3 a serum levels and $\beta$-catenin tissue expression, and found that high wnt3a serum levels and $\beta$-catenin overexpression were correlated with poor prognosis. Importantly, our study showed that serum levels of wnt3a with a cut-off value of $25 \mathrm{ng} / \mathrm{ml}$ were a good predictor for EC early diagnosis. Positive $\beta$-catenin expression was important in predicting prognosis. Kaplan-Meier analysis indicated that patients with higher serum levels of wnt3a and $\beta$-catenin expression were more likely to have a poor prognosis, supporting that the wnt $/ \beta$-catenin pathway is important for predicting EC prognosis. But the cox regression model showed that the $R R$ value of wnt3a (RR, 2.427; 95\% confidence interval, 0.521-11.295, $P=$ $0.258)$ was not statically significant, and the OR value (10.551, $95 \%$ confidence interval, $2.708-41.111, P=0.001$ ) was lower than that of $\operatorname{cox} 2$ serum levels. Therefore, we speculated that cox 2 might regulate EC growth and metastasis via the wnt $/ \beta$-catenin pathway and could function as a potential predictive biomarker for EC diagnosis and prognosis. Detecting cox2 serum levels might thus conveniently and efficiently aid early diagnosis of EC and prognosis prediction. As $\beta$-catenin expression was also a risk factor for prognosis, combined the serum levels of cox 2 with $\beta$-catenin expression, the predictive value could be enhanced.

This study had several limitations as well. Firstly, due to the currently limited experimental technology, we only detected the expression levels of $\operatorname{cox} 2$ and wnt pathway-related proteins using immunochemistry and ELISA assays and assessed the correlation between these biomarkers and the diagnosis and prognosis of EC based on these results. However, the detailed molecular regulatory mechanisms of the action of these proteins in EC were not delineated or verified through in vitro or in vivo experiments; these aspects require further investigation. What's more, this study only included the Asiatic population and endometrioid histology, which may cause some limitation in generalizing the conclusion. Additionally, in this study, we included some young patients, which might have some genetic influence and bias. So, the age range scope could be reduced and microsatellite instability related proteins should be detected to excluded individuals with systemic predisposition to cancer like lynch syndrome. In addition, as this is an ongoing prospective study, the sample size and follow up time have been limited up to now. The sensitivity was high but specificity was lower, moreover, the overall survival time for patients did not reveal a significant difference, the reliability and validity of the cut-off value were not evaluated as well. Further big sample size, multi-center, randomly clinical controlled trials are still needed.

\section{CONCLUSION}

In conclusion, our study showed that $\operatorname{cox} 2$ and $\beta$-catenin were overexpressed in EC tissues and the serum levels of cox 2 and wnt $3 a$ in patients with EC were elevated as well. Cox 2 serum level and $\beta$-catenin expression were positively correlated with EC diagnosis and poor prognosis. Cox 2 and $\beta$-catenin might potentially function as a predictable biomarkers for EC patients. This study had certain limitations, but provides a basis for further exploration into the mechanisms of action of cox2 in EC and its potential clinical application as a diagnostic and prognostic marker.

\section{DATA AVAILABILITY STATEMENT}

The datasets used and analyzed during the current study are available from the corresponding author on reasonable request.

\section{ETHICS STATEMENT}

The studies involving human participants were reviewed and approved by the Medical Ethics Committee of the China Japan Friendship Hospital. The patients/participants provided their written informed consent to participate in this study.

\section{AUTHOR CONTRIBUTIONS}

LD carried out the experiments and wrote the manuscript. All authors analyzed the experimental results and revised the manuscript.

\section{FUNDING}

This work was supported by the National Natural Science Foundation of China (Grant No. 81372777) and by the Fundamental Research Funds for the Central Universities and Research Projects on Biomedical Transformation of China-Japan Friendship Hospital (Grant No. PYBZ1820). 


\section{ACKNOWLEDGMENTS}

The authors would like to thank the patient for agreeing and providing her case history and thank the National Natural Science Foundation of China and China-Japan Friendship Hospital for funding.

\section{REFERENCES}

1. Weiderpass E, Labrèche F. Malignant tumors of the female reproductive system. Saf Health Work. (2012) 3:166-80. doi: 10.5491/SHAW.2012.3.3.166

2. Liu $\mathrm{Y}$, Li H, Zhao C, Jia H. MicroRNA-101 inhibits angiogenesis via COX-2 in endometrial carcinoma. Mol Cell Biochem. (2018) 448:61-9. doi: 10.1007/s11010-018-3313-0

3. Zhang H, Wang S, Cacalano N, Zhu H, Liu Q, Xie M, et al. Oncogenic Y68 frame shift mutation of PTEN represents a mechanism of docetaxel resistance in endometrial cancer cell lines. Sci Rep. (2019) 9:2111. doi: 10.1038/s41598-019-38585-9

4. van der Zee M, Jia Y, Wang Y, Heijmans-Antonissen C, Ewing PC, Franken $\mathrm{P}$, et al. Alterations in Wnt- $\beta$-catenin and Pten signalling play distinct roles in endometrial cancer initiation and progression. J Pathol. (2013) 230:48-58. doi: $10.1002 /$ path.4160

5. Li W, Xu RJ, Zhang HH, Jiang LH. Overexpression of cyclooxygenase-2 correlates with tumor angiogenesis in endometrial carcinoma. Int J Gynecol Cancer. (2006) 16:1673-8. doi: 10.1111/j.1525-1438.2006.00408.x

6. Cai S, Zhang YX, Han K, Ding YQ. Expressions and clinical significance of COX-2, VEGF-C, and EFGR in endometrial carcinoma. Arch Gynecol Obstet. (2017) 296:93-8. doi: 10.1007/s00404-017-4386-9

7. Moreno-Bueno G, Hardisson D, Sánchez C, Sarrió D, Cassia R, García-Rostán $\mathrm{G}$, et al. Abnormalities of the APC/beta-catenin pathway in endometrial cancer. Oncogene. (2002) 21:7981-90. doi: 10.1038/sj.onc.1205924

8. Lambropoulou M, Alexiadis G, Limberis V, Nikolettos N, Tripsianis G. Clinicopathologic and prognostic significance of cyclooxygenase-2 expression in endometrial carcinoma. Histol Histopathol. (2005) 20:753-9. doi: 10.14670/HH-20.753

9. Daikoku T, Terakawa J, Hossain MM, Yoshie M, Cappelletti M, Yang P, et al. Mammalian target of rapamycin complex 1 and cyclooxygenase 2 pathways cooperatively exacerbate endometrial cancer. Am J Pathol. (2014) 184:2390-402. doi: 10.1016/j.ajpath.2014.05.023

10. Zhang J, Luo J, Ni J, Tang L, Zhang HP, Zhang L, et al. MMP-7 is upregulated by COX-2 and promotes proliferation and invasion of lung adenocarcinoma cells. Eur J Histochem. (2014) 58:2262. doi: 10.4081/ejh.2014.2262

11. Fukazawa EM, Baiocchi G, Soares FA, Kumagai LY, Faloppa CC, BadiglianFilho L, et al. Cox-2, EGFR, and ERBB-2 expression in cervical intraepithelial neoplasia and cervical cancer using an automated imaging system. Int J Gynecol Pathol. (2014) 33:225-34. doi: 10.1097/PGP.0b013e318290405a

12. Kim SH, Ahn BK, Paik SS, Lee KH. Cyclooxygenase- 2 expression is a predictive marker for late recurrence in colorectal cancer. Gastroenterol Res Pract. (2018) 2018:7968149. doi: 10.1155/2018/7968149

13. Ferrandina G, Legge F, Ranelletti FO, Zannoni GF, Maggiano N, Evangelisti A, et al. Cyclooxygenase-2 expression in endometrial carcinoma: correlation with clinicopathologic parameters and clinical outcome. Cancer. (2002) 95:801-7. doi: 10.1002/cncr.10736

14. Ahmed M, Hussain AR, Siraj AK, Uddin S, Al-Sanea N, Al-Dayel F, et al. Co-targeting of cyclooxygenase-2 and FoxM1 is a viable strategy in inducing anticancer effects in colorectal cancer cells. Mol Cancer. (2015) 14:131. doi: 10.1186/s12943-015-0406-1

15. García Mitacek MC, Praderio RG, Stornelli MC, de la Sota RL, Stornelli MA. Prostaglandin synthesis enzymes' gene transcription in bitches with endometritis. Reprod Domest Anim. (2017) 52:298-302. doi: $10.1111 /$ rda. 12950

16. Herschman HR. Primary response genes induced by growth factors and tumor promoters. Annu Rev Biochem. (1991) 60:281-319. doi: 10.1146/annurev.bi.60.070191.001433

\section{SUPPLEMENTARY MATERIAL}

The Supplementary Material for this article can be found online at: https://www.frontiersin.org/articles/10.3389/fonc. 2020.00056/full\#supplementary-material

17. Clevers H. Wnt/beta-catenin signaling in development and disease. Cell (2006) 127:469-80. doi: 10.1016/j.cell.2006.10.018

18. Schlosshauer PW, Pirog EC, Levine RL, Ellenson LH. Mutational analysis of the CTNNB1 and APC genes in uterine endometrioid carcinoma. Mod Pathol. (2000) 13:1066-71. doi: 10.1038/modpathol.3880196

19. McConechy MK, Ding J, Senz J, Yang W, Melnyk N, Tone AA, et al. Ovarian and endometrial endometrioid carcinomas have distinct CTNNB1 and PTEN mutation profiles. Mod Pathol. (2014) 27:128-34. doi: 10.1038/modpathol.2013.107

20. Hou X, Tan Y, Li M, Dey SK, Das SK. Canonical Wnt signaling is critical to estrogen-mediated uterine growth. Mol Endocrinol. (2004) 18:3035-49. doi: 10.1210/me.2004-0259

21. Wang Y, Hanifi-Moghaddam P, Hanekamp EE, Kloosterboer HJ, Franken P, Veldscholte J, et al. Progesterone inhibition of Wnt/beta-catenin signaling in normal endometrium and endometrial cancer. Clin Cancer Res. (2009) 15:5784-93. doi: 10.1158/1078-0432.CCR-09-0814

22. Sato A, Yamamoto H, Sakane H, Koyama H, Kikuchi A. Wnt5a regulates distinct signalling pathways by binding to Frizzled2. Embo J. (2010) 29:41-54. doi: 10.1038/emboj.2009.322

23. Simons M, Mlodzik M. Planar cell polarity signaling: from fly development to human disease. Annu Rev Genet. (2008) 42:517-40. doi: 10.1146/annurev.genet.42.110807.091432

24. De Craene B, Berx G. Regulatory networks defining EMT during cancer initiation and progression. Nat Rev Cancer. (2013) 13:97-110. doi: $10.1038 /$ nrc3447

25. Knific T, Osredkar J, Smrkolj Š, Tonin I, Vouk K, Blejec A, et al. Novel algorithm including CA-125, HE4 and body mass index in the diagnosis of endometrial cancer. Gynecol Oncol. (2017) 147:126-32. doi: 10.1016/j.ygyno.2017.07.130

26. Krajewska M, Krajewski S, Epstein JI, Shabaik A, Sauvageot J, Song K, et al. Immunohistochemical analysis of bcl-2, bax, bcl-X, and mcl-1 expression in prostate cancers. Am J Pathol. (1996) 148:1567-76.

27. Kahane H, Sharp JW, Shuman GB, Dasilva G, Epstein JI. Utilization of high molecular weight cytokeratin on prostate needle biopsies in an independent laboratory. Urology. (1995) 45:981-6. doi: 10.1016/S0090-4295(99)80118-6

28. Zhou B, Pan Y, Yu Q, Zhai Z. Fibrinogen facilitates atherosclerotic formation in Sprague-Dawley rats: a rodent model of atherosclerosis. Exp Ther Med. (2013) 5:730-4. doi: 10.3892/etm.2013.913

29. Kang B, Jiang D, He H, Ma R, Chen Z, Yi Z. Effect of Oazl overexpression on goose ovarian granulosa cells. Amino Acids. (2017) 49:1123-32. doi: 10.1007/s00726-017-2411-8

30. Sohail ML, Khan MS, Ijaz M, Naseer O, Fatima Z, Ahmad AS, et al. Seroprevalence and risk factor analysis of human leptospirosis in distinct climatic regions of Pakistan. Acta Trop. (2018) 181:79-83. doi: 10.1016/j.actatropica.2018.01.021

31. Dobrzycka B, Mackowiak-Matejczyk B, Kinalski M, Terlikowski SJ. Pretreatment serum levels of bFGF and VEGF and its clinical significance in endometrial carcinoma. Gynecol Oncol. (2013) 128:454-60. doi: 10.1016/j.ygyno.2012.11.035

32. Yamazawa K, Shimada H, Hirai M, Hirashiki K, Ochiai T, Ishikura H, et al. Serum p53 antibody as a diagnostic marker of high-risk endometrial cancer. Am J Obstet Gynecol. (2007) 197:505.e501-7. doi: 10.1016/j.ajog.2007. 04.033

33. Stiekema A, Lok C, Korse CM, van Driel WJ, van der Noort V, Kenter GG, et al. Serum HE4 is correlated to prognostic factors and survival in patients with endometrial cancer. Virchows Arch. (2017) 470:655-64. doi: 10.1007/s00428-017-2115-1 
34. Steinbakk A, Gudlaugsson E, Aasprong OG, Skaland I, Malpica A, Feng W, et al. Molecular biomarkers in endometrial hyperplasias predict cancer progression. Am J Obstet Gynecol. (2011) 204:357.e351-7.312. doi: 10.1016/j.ajog.2010.12.007

35. Chiu HC, Li CJ, Yiang GT, Tsai AP, Wu MY. Epithelial to mesenchymal transition and cell biology of molecular regulation in endometrial carcinogenesis. J Clin Med. (2019) 8:E439. doi: 10.3390/jcm8040439

36. Mehdinejadiani S, Amidi F, Mehdizadeh M, Barati M, Safdarian $\mathrm{L}$, Aflatoonian $\mathrm{R}$, et al. The effects of letrozole and clomiphene citrate on ligands expression of Wnt3, Wnt7a, and Wnt8b in proliferative endometrium of women with Polycystic ovarian syndrome. Gynecol Endocrinol. (2018) 34:775-80. doi: 10.1080/09513590.2018. 1446934

37. Anastas JN, Moon RT. WNT signalling pathways as therapeutic targets in cancer. Nat Rev Cancer. (2013) 13:11-26. doi: 10.1038/nrc3419

38. Anastas JN. Functional crosstalk between WNT signaling and tyrosine kinase signaling in cancer: Seminars in Oncology, Vol 42, No 6, December 2015, pp 820-831. Semin Oncol. (2016) 43:526. doi: 10.1053/j.seminoncol.2016. 06.010

39. Eskander RN, Ali S, Dellinger T, Lankes HA, Randall LM, Ramirez NC, et al. Expression patterns of the Wnt pathway inhibitors Dickkopf3 and secreted frizzled-related proteins 1 and 4 in endometrial endometrioid adenocarcinoma: an NRG oncology/Gynecologic Oncology Group Study. Int J Gynecol Cancer. (2016) 26:125-32. doi: 10.1097/IGC.0000000000000563

40. Humphries AC, Mlodzik M. From instruction to output: Wnt/PCP signaling in development and cancer. Curr Opin Cell Biol. (2018) 51:110-6. doi: 10.1016/j.ceb.2017.12.005

41. Tan DS, Holzner M, Weng M, Srivastava Y, Jauch R. SOX17 in cellular reprogramming and cancer. Semin Cancer Biol. (2019). doi: 10.1016/j.semcancer.2019.08.008. [Epub ahead of print].
42. Chen JJ, Xiao ZJ, Meng X, Wang Y, Yu MK, Huang WQ, et al. MRP4 sustains Wnt/ $\beta$-catenin signaling for pregnancy, endometriosis and endometrial cancer. Theranostics. (2019) 9:5049-64. doi: 10.7150/thno.32097

43. Scholten AN, Creutzberg CL, van den Broek LJ, Noordijk EM, Smit VT. Nuclear beta-catenin is a molecular feature of type I endometrial carcinoma. $J$ Pathol. (2003) 201:460-5. doi: 10.1002/path.1402

44. Saegusa M, Hashimura M, Yoshida T, Okayasu I. Beta-catenin mutations and aberrant nuclear expression during endometrial tumorigenesis. Br J Cancer. (2001) 84:209-17. doi: 10.1054/bjoc.2000.1581

45. Goad J, Ko YA, Kumar M, Jamaluddin MFB, Tanwar PS. Oestrogen fuels the growth of endometrial hyperplastic lesions initiated by overactive Wnt/ $\beta$-catenin signalling. Carcinogenesis. (2018) 39:1105-16. doi: 10.1093/carcin/bgy079

46. Liu Y, Patel L, Mills GB, Lu KH, Sood AK, Ding L, et al. Clinical significance of CTNNB1 mutation and Wnt pathway activation in endometrioid endometrial carcinoma. J Natl Cancer Inst. (2014) 106:dju245. doi: 10.1093/jnci/dju245

47. Coopes A, Henry CE, Llamosas E, Ford CE. An update of Wnt signalling in endometrial cancer and its potential as a therapeutic target. Endocr Relat Cancer. (2018) 25:R647-62. doi: 10.1530/ERC-18-0112

Conflict of Interest: The authors declare that the research was conducted in the absence of any commercial or financial relationships that could be construed as a potential conflict of interest.

Copyright (C) 2020 Deng, Liang and Han. This is an open-access article distributed under the terms of the Creative Commons Attribution License (CC BY). The use, distribution or reproduction in other forums is permitted, provided the original author(s) and the copyright owner(s) are credited and that the original publication in this journal is cited, in accordance with accepted academic practice. No use, distribution or reproduction is permitted which does not comply with these terms. 